\title{
Ferroelectric properties of a triphenylene derivative with polar functional groups in the crystalline state
}

\author{
Atsushi Sugita, Kyoko Suzuki, and Shigeru Tasaka* \\ Department of Materials Science and Chemical Engineering, Shizuoka University, 3-5-1 Johoku, Hamamatsu, \\ Shizuoka 432-8561, Japan
}

(Received 2 February 2004; published 16 June 2004)

\begin{abstract}
We studied ferroelectric ordering in a triphenylene derivative embedded with electric dipoles $[2,3,6,7,10,11$ hexakis (4-octyloxy-benzoyloxy) triphenylene (HOBPT)] in a crystalline state. Experimental results indicate that the ferroelectricity in HOBPT is caused by an ordered orientation of $\mathrm{C}=\mathrm{O}$ dipoles. Our experiments also reveal that dielectric anomaly due to ferroelectric-paraelectric phase transition occurs at $380 \mathrm{~K}$. A photovoltaic effect was observed in an electrically treated thin film of HOBPT. The phenomenon results from a high charge mobility due to the $\pi-\pi$ stack between adjacent molecules as well as an internal electric field derived by the residual polarization.
\end{abstract}

DOI: 10.1103/PhysRevB.69.212201

PACS number(s): 77.80.Bh, 72.40.+w

Ferroelectric materials have attracted great interest among researchers owing to their large dielectric, piezoelectric, and pyroelectric coefficients, and they are applied to several electric devices such as capacitors, piezoelectric vibrators, or pyroelectric infrared sensors. ${ }^{1-4}$ Great efforts are being made to develop a new type of thin film ferroelectric memory, taking advantage of field-induced polarization inversion. 5 These materials are also interesting regarding their optical functionality, such as nonlinear optical effect or electro-optical effect. ${ }^{6}$ Until now, many inorganic ferroelectric materials, such as $\mathrm{BaTiO}_{3}$ and $\mathrm{KH}_{2} \mathrm{PO}_{4}$, have been developed and applied for electrical and optical devices. ${ }^{1-3}$ Several organic ferroelectric crystals were also designed and proposed, such as thiourea and phenanthrene. Ferroelectricity is not unique to crystalline materials, and it has been reported even for noncrystal organic materials, namely liquid crystals, crystalline, and amorphous polymers. ${ }^{7}$

In the present report, we propose a type of organic ferroelectric materials based on a triphenylene derivative embedded with dipoles [2,3,6,7,10,11-hexakis(4-octyloxybenzoyloxy) triphenylene (HOBPT) (Fig. 1)] in a crystalline state. We also report the photovoltaic effect in electrically treated HOBPT. The main body of HOBPT consists of the core triphenylene ring substituted by six carbonyl $(\mathrm{C}=\mathrm{O})$ groups, which have dipole moments. Each carbonyl group is further connected to a moderately long alkyl group with a flexible structure. A series of triphenylene derivatives have been investigated extensively because they exhibit high carrier mobility in both crystalline and liquid-crystalline states. $^{8-11}$ The flat faces of disk-like triphenylene rings were self-assembled and they form one-dimensional columns. Since the $\pi$ electron orbits are overlapped between the adjacent molecules in the columns, the carriers are allowed to transport along the columnar axis. It was reported previously that $(2,3,6,7,10,11$-hexahexyl) thiotriphenylene exhibited a charge-carrier mobility of the order of as high as $0.1 \mathrm{~cm}^{2} \mathrm{~V}^{-1} \mathrm{~s}^{-1}$ in a highly ordered columnar liquid crystalline phase. ${ }^{8}$ Recently, a photovoltaic effect was found in a mixture of discotic molecules and dye molecules. ${ }^{12}$ Because of a hetero-junction between the discotic molecules and the dye molecules, photo-injected electron-hole pairs in the dye molecules were dissociated. This leads to transportation of the photo carriers along the highly conducting column. However, there have been no reports about the ferroelectricity in the groups of triphenylene derivatives. Furthermore, a photovoltaic effect found in the HOBPT system stemmed from a mechanism completely different from the former case in the discotic molecule/dye molecule complex.

HOBPT was synthesized with a method previously reported. ${ }^{13}$ The powder of HOBPT was dissolved in methanol, and the solution was cast and dried on an aluminum substrate to form a thin film sample. A thin layer of aluminum was deposited on the other side of the film with a vacuum evaporation method. Four phase-transition temperatures were observed between 270 and 520 $\mathrm{K}$ from a differential calorimetric scanning measurement. The phase sequence is in a good agreement with that re-

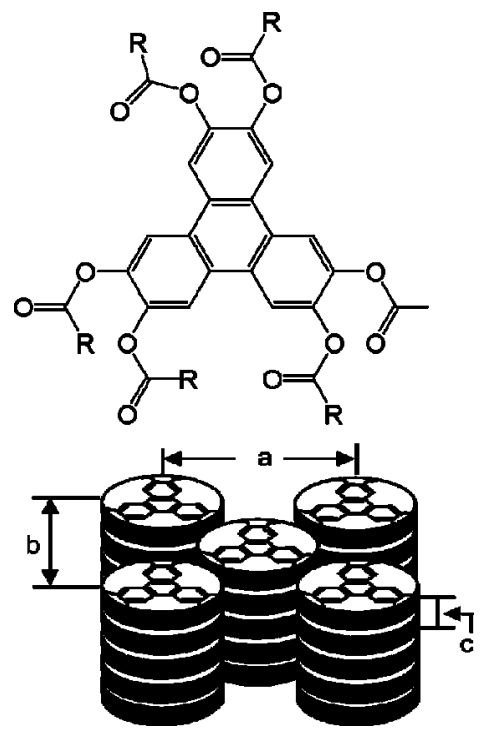

FIG. 1. Chemical structure of 2,3,6,7,10,11-hexakis(4-octyloxybenzoyloxy) triphenylene (HOBPT) and a schematic view of crystalline HOBPT. 


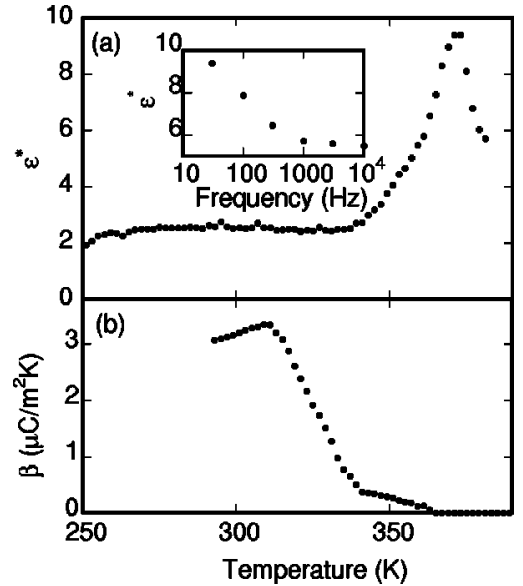

FIG. 2. (a) Temperature dependence of dielectric constant $\epsilon^{*}$ of HOBPT. The frequency dependence of $\epsilon^{*}$ at the dielectric anomaly point is also shown in the inset. (b) Temperature dependence of pyroelectric coefficient $\beta$ of HOBPT.

ported in a previous report; ${ }^{14}$ crystalline (I) phase/ $(383 \mathrm{~K}) /$ crystalline (II) phase/(426 K)/columnar liquid crystalline phase $/(438 \mathrm{~K}) /$ discotic nematic crystalline phase / $(509 \mathrm{~K}) /$ isotropic phase. The present work was concentrated on the electric and optical properties of HOBPT in the crystalline phase. All the measurement was performed at the temperatures lower than $400 \mathrm{~K}$, where the molecular form of crystalline phases appear.

A wide-angle X-ray scattering (WAXS) pattern was studied to investigate a crystal structure of HOBPT at $293 \mathrm{~K}$ (Rigaku-Rad B diffractometer, CuKa $1.542 \AA, 20 \mathrm{kV}$, $20 \mathrm{~mA}$ radiation). Two intense sharp peaks were observed at $2 \theta=3.50$ and $3.86^{\circ}$ in the WAXS pattern. This indicates that the crystal structure of HOBPT was preferably assigned to an orthorhombic structure and it was similar to the discotic rectangular liquid crystalline mesophase. Some of the other triphenylene derivatives were reported to constitute a closepacked hexagonal structure. ${ }^{15,16}$ In the formation of a hexagonal structure, however, a single sharp peak should appear, instead of the double peaks. A broad peak was also seen at around $\theta=20^{\circ}$. The existence of the broad peak indicates that the assembly of HOBPT molecules forms a polycrystalline structure and that some portions of the triphenylene groups are stacked irregularly. This may be because the structurally flexible alkyl groups with flexible structure avoid close packing between the adjacent triphenylene groups. This result suggests that the side groups of HOBPT, including $\mathrm{C}=\mathrm{O}$ groups, are allowed to move freely to some extent even in the crystalline phase.

Two experiments were performed to demonstrate ferroelectricity in crystalline HOBPT. First, dielectric constant $\left(\epsilon^{*}\right)$ was investigated at several temperatures $(T)$, using the LCR meter (4232A, Hewlett Packard). Figure 2 is a $T-\epsilon^{*}$ curve measured at the external field frequency of $30 \mathrm{~Hz}$. A dielectric anomaly, corresponding to the ferroelectric-paraelectric phase transition, is found at $T_{c}$ $=370 \mathrm{~K}$. The $T-\epsilon^{*}$ curve was studied at other frequencies from 30 to $10 \mathrm{kHz}$. Although the dielectric anomaly was observed at about $370 \mathrm{~K}$ over the entire frequency range
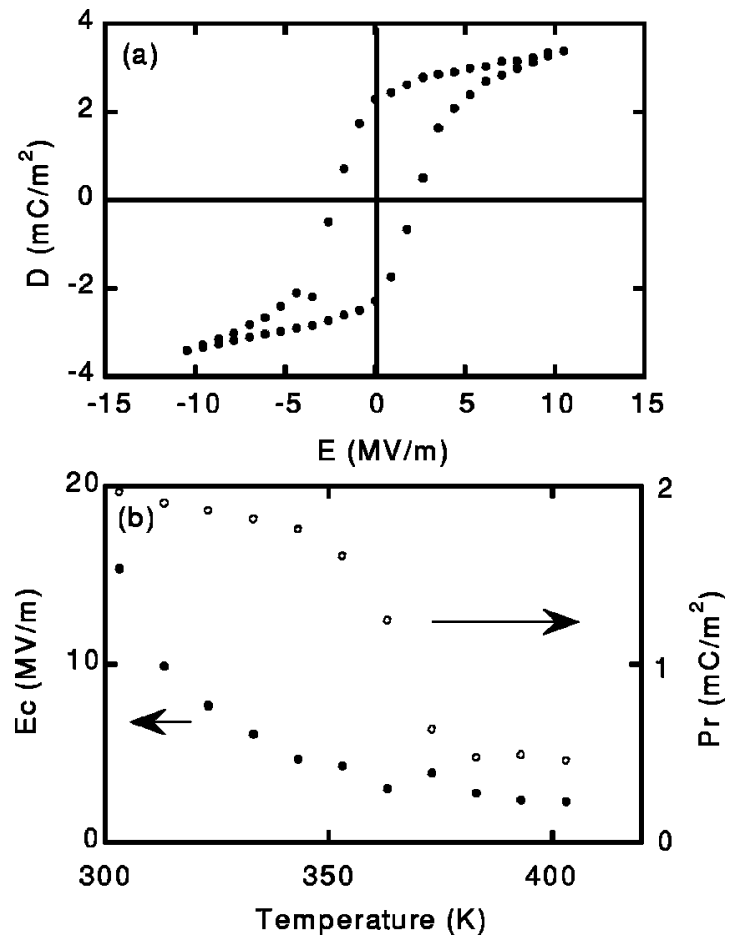

FIG. 3. (a) Electric field $(E)$-electric displacement $(D)$ hysteresis loop of HOBPT at $293 \mathrm{~K}$. (b) Temperature dependence of coercive field $\left(E_{c}\right)$ and residual polarization $\left(P_{r}\right)$ of HOBPT.

measured, the magnitude of $\epsilon^{*}$ decreases with the increment of the field frequency (an inset in Fig. 2). A pyroelectric coefficient $\beta$ was also measured as a function of $T$. The coefficient is defined as a temperature derivative of the macroscopic polarization. The observation of the pyroelectricity indicates indirectly the formation of the macroscopic polarization in the materials. The magnitude of $\beta$ was recorded with a method previously reported. ${ }^{17}$ The sample was poled with a DC external field of $20 \mathrm{MV} / \mathrm{m}$ at $350 \mathrm{~K}$ before the measurement of $\beta$. The quantity of $\beta$ shows a gradual increase up to $310 \mathrm{~K}$, above which it decreases and approaches a zero level at around $370 \mathrm{~K}$. This results also claims that the macroscopic polarization exists at the temperatures lower than the phase transition point.

Second, the electric-field $(E)$ dependence of an electric displacement $(D)$ was studied. The current $(I)$ - electric field $(E)$ hysteresis loop was measured using a high-voltage power supply and an electrometer. Triangular input voltage of $0.03 \mathrm{~Hz}$ in frequency was applied between the aluminum electrodes. The $D-E$ hysteresis loop was derived by integrating $I$ as a function of $E$. Figure 3(a) shows the $D-E$ hysteresis loop of HOBPT at $293 \mathrm{~K}$. The coercive electric field $\left(E_{c}\right)$ and residual polarization $\left(P_{r}\right)$ were determined to be $10.3 \mathrm{MV} / \mathrm{m}$ and $2.0 \mathrm{mC} / \mathrm{m}^{2}$, respectively. $E_{c}$ and $P_{r}$ at several temperatures are shown in Fig. 3(b). Residual polarization decreases gradually as temperature rises up to $380 \mathrm{~K}$ and approaches to a nearly constant value $\sim 0.5 \mathrm{mC} / \mathrm{m}^{2}$ at the temperatures above $383 \mathrm{~K}$. This nonzero residual polarization at $T>T_{c}$ might be due to space charge polarization or spontaneous polarization stably existing in the crystalline (II) phase. 


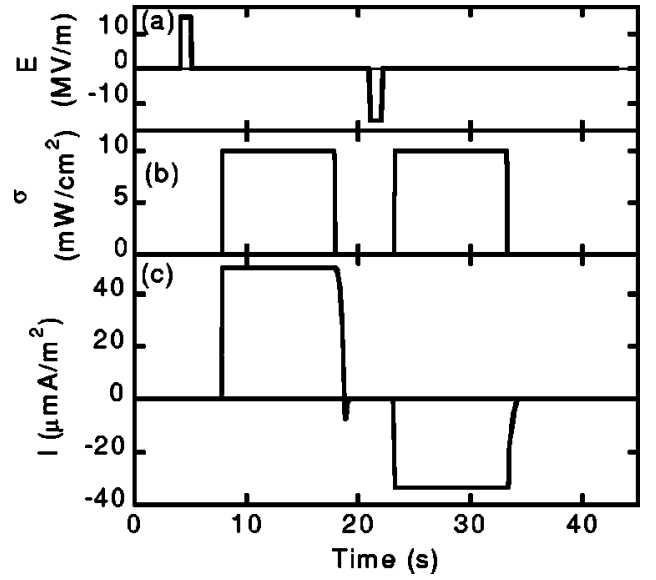

FIG. 4. Examples of photovoltaic effects of poled HOBPT in a short circuit. A step wise application of DC external electric field (a), irradiation of UV light (b) and a photo-current response (c) are shown.

HOBPT has six polar $\mathrm{C}=\mathrm{O}$ groups and each of them has an electric dipole moment of about 2.3 Debye. ${ }^{18}$ According to a molecular orbital calculation with an AM1 method, the dipole moment of the HOBPT molecule in an isolated state was estimated to be 1.8 Debye and its direction was perpendicular to the flat face of the core triphenylene rings. The residual polarization of $P_{r}=2.0 \mathrm{mC} / \mathrm{m}^{2}$ at $293 \mathrm{~K}$ is equivalent to the dipole moment of 1.7 Debye per molecule. Here, a weight density of about $1 \mathrm{~g} / \mathrm{cm}^{3}$, which was determined with a float sink method, was used for the estimation. Thus, the dipole moment in each molecule may be highly oriented along the columnar axis, and the macroscopic polarization was formed in the direction. The dielectric constant $\epsilon^{*}$ was dispersive in the frequency range measured in the present study. This low-frequency dielectric relaxation may be due to the slowly developing rotational motion of the dipoles in response to the external field. A similar slow dielectric relaxation was observed for a ferroelectric liquid crystal embedded with carbonyl groups. ${ }^{19}$ As for the polarization inversion found in the $D-E$ hysteresis, a relatively large free volume should be necessary for the dipoles to rotate inside the crystal. As characterized by the XRD pattern, the HOBPT system constitutes the orthorhombic structure, not the close-packed hexagonal structure. Furthermore, parts of the triphenylene rings were stacked irregularly along the columnar axis. The partial irregularity in the intramolecular and intermolecular aggregation accounts for the free volume allowing the dipole orientation.

Next, the anomalous photovoltaic effect in the poled HOBPT is discussed. The photovoltaic effect was demonstrated with a procedure mentioned as follows (Fig. 4). First, the DC external field was applied to the HOBPT sample sandwiched between the aluminum electrodes for $1 \mathrm{~s}$. One of the aluminum electrodes is thin enough to transmit UV light. After turning off the field, UV light from a high-pressure mercury lamp $\left(1 \mathrm{~mW} / \mathrm{cm}^{2}\right)$ was irradiated on the electrically poled sample for $10 \mathrm{~s}$. Then the irradiation of the UV light was turned off. Second, the DC external field with an inverse direction was applied for $1 \mathrm{~s}$. The UV light was irradiated on the sample for another $10 \mathrm{~s}$. The electric current in a short circuit was monitored during a series of the field application and the UV-light irradiation. All the experiment was performed at $293 \mathrm{~K}$. No bias voltage was applied to the sample during the irradiation of the UV light.

The current was recorded during the irradiation of the UV light. The magnitude of about $50 \mu \mathrm{A} / \mathrm{m}^{2}$ was kept nearly constant level during the UV light irradiation. When the electric field with an inverse direction was applied, the photoinduced current with an inverse direction was recorded. After a series of the poling and the UV light irradiation, the sample was heated up to $400 \mathrm{~K}$, and then cooled down to be crystallized again. This time, no photo-induced current was recorded. This experiment was executed using a thin film sample sandwiched between electrodes made of indium tin oxide (ITO) and silver. The photovoltaic effect was observed, independent of the kinds of the electrodes. In contrast, the photo-induced current was not recorded at $380 \mathrm{~K}$, the temperature higher than $T_{c}$.

This phenomenon appears to be analogous to the photovoltaic effect observed in a $\mathrm{p}-\mathrm{n}$ junction type semiconductor or Shottkey junction on a metal-semiconductor surface. ${ }^{2,20}$ The photo-induced current observed in Shottkey-type photovoltaic effect is dependent sensitively on the type of the electrodes. In this study, however, such metal type dependence was not clearly recognized. The present photovoltaic effect should be different from these conventional ones in that the direction of the photo-induced current is reversible depending on the polarity of the applied field. The triggering mechanism of the photovoltaic effect is closely related to the existence of the residual polarization. In fact, the significant level of the photo-induced current was not detected at temperatures higher than $T_{c}$, where pyroelectric signal was not observed. It should be referred to as an anomalous photovoltaic effect, as was reported in inorganic ferroelectric materials like $\mathrm{LiNbO}_{3}$ and $\mathrm{BaTiO}_{3}$ as well as polymer-based ferroelectric materials such as PVDF. ${ }^{21,22}$

A following mechanism has been proposed to explain the anomalous photovoltaic effect in ferroelectric materials. ${ }^{21}$ The molecules are led to a charged excited state upon photoexcitation, with the consequence that the charged carriers are generated. An internal electric field induced by the residual polarization triggers the transfer of these photo-carriers between the neighboring sites. When the direction of the residual polarization is reversed according to the external field, the polarity of the photo-induced voltage is also reversed. Thus, a high charge mobility and large residual polarization are necessary conditions for the anomalous photovoltaic effect. The electrically poled HOBPT system satisfies these conditions; the higher charge mobility is realized in the onedimensional columns consisting of the self-assembled triphenylene groups. In the columns, the conjugation of the $\pi$ orbital between the adjacent molecules allows the photocarriers to transport. The triphenylene derivatives in the liquid-crystalline meso phases typically have a hole carrier mobility on the order of $10^{-3}-10^{-2} \mathrm{~cm}^{2} \mathrm{~V}^{-1} \mathrm{~s}^{-1} \cdot{ }^{8,9} \mathrm{HOBPT}$ in the present study should also have the same order of the mobility as other triphenylene derivatives, taking into account that it constructs the similar structure. In contrast, the observation of the $D-E$ hysteresis and the pyroelectric sig- 
nal show that the macroscopic polarization exits in the ferroelectric phase of the HOBPT system, as mentioned above. The macroscopic polarization introduces the internal field in the system. Assuming that the internal electric field of $P_{r} / \epsilon_{0}(\epsilon-1) \sim 140 \mathrm{MV} / \mathrm{m}$ was applied in the direction along the columnar axis inthe sample with $50 \mu \mathrm{m}$ thickness, it follows that the light energy converted into the photo-carrier was $50 \mu \mathrm{A} / \mathrm{m}^{2} \times 140 \mathrm{MV} / \mathrm{m} \times 50 \mu \mathrm{m}=47 \mu \mathrm{W} / \mathrm{cm}^{2}$. The energy conversion rate is estimated to be about $0.5 \%$.

Finally, we comment on a slowly decreasing tail in the photo-induced current that appears upon turning off the UVlight irradiation. The signal may be due to pyroelectric signal, which is induced by a modulation of the residual polarization against the temperature change in the crystal. When the UV light irradiation was turned off, HOBPT begins to emit a heat stored during the light irradiation. The diffusion of the heat introduces the temperature change around the crystal surface, which induces the pyroelectric signal.

In conclusion, the electric properties of the triphenylene derivative, HOBPT, were investigated from the $T-\epsilon^{*}$ curve, the $T-\beta$ curve and the $D-E$ hysteresis. These experimental results show the ferroelectric ordering in HOBPT in the crystalline state. Furthermore, the anomalous photovoltaic effect observed in electrically poled HOBPT is caused by the intracolumnar charge carrier transfer and the internal field due to the residual polarization.

The authors thank Dr. Masaki Takesada and Dr. Shigehiro Takasaka for fruitful discussions and suggestions and Dr. Seiji Sugita for his instruction about English presentation.
*Electronic mail: tcstasa@ipc.shizuoka.ac.jp

${ }^{1}$ B. A. Strukov and A. P. Levanyuk, Ferroelectric Phenomena in Crystals: Physical Foundations (Springer-Verlag, Berlin, 1998).

${ }^{2}$ C. Kittel, Introduction to Solid State Physics, 7th ed. (Wiley, New York, 1996).

${ }^{3}$ M. E. Lines and A. M. Glass, Principles and Applications of Ferroelectrics and Related Materials (Oxford University Press, Oxford, 2001).

${ }^{4}$ K. Uchino, Ferroelectric Devices Vol. 16-Materials Engineering (Marcel Dekker, New York, 2001).

${ }^{5}$ J. F. Scott, Ferroelectric Memories (Springer-Verlag, Berlin, 2000), Vol. 3.

${ }^{6}$ V. G. Dmitriev, G. G. Gruzadyan, and D. N. Nikogosyan, Handbook of Nonlinear Optical Crystals, 2nd ed. (Springer-Verlag, Berlin, 1997).

${ }^{7}$ H. S. Nalwa, Ferroelectric Polymers: Chemistry, Physics, and Applications. Vol. 28-Plastics Engineering (Marcel Dekker, New York, 1995).

${ }^{8}$ D. Adam, P. Schuhmacher, J. Simmerer, L. Häussling, K. Simmensmeyer, K. H. Etzbach, H. Ringsdorf, and D. Haarer, Nature (London) 371, 141 (1994).

${ }^{9}$ N. Boden, R. J. Bushby, J. Clements, B. Movaghar, K. J. Donovan, and T. Kreouzis, Phys. Rev. B 52, 13274 (1995).

${ }^{10}$ A. M. van de Craats, L. D. A. Siebbeles, I. Bleyl, D. Haarer, Y. A. Berlin, A. A. Zharikov, and J. M. Warman, J. Phys. Chem. B 102, 9625 (1998).
${ }^{11}$ B. R. Wegewijis, L. D. A. Siebbeles, N. Boden, R. J. Bushby, B. Movaghar, O. R. Lozman, Q. Liu, A. Pecchia, and L. A. Mason, Phys. Rev. B 65, 245112 (2002).

${ }^{12}$ L. Schmidt-Mende, A. Fechtenkotter, K. Mullen, E. Moons, R. H. Friend, and J. D. MacKenzie, Science 293, 1119 (2001).

${ }^{13}$ J. A. Rego, S. Kumar, and H. Ringsdorf, Chem. Mater. 8, 1402 (1996).

${ }^{14}$ N. H. Tinh, H. Gasparouz, and C. Destrate, Mol. Cryst. Liq. Cryst. 68, 101 (1981).

${ }^{15}$ G. B. M. Vaughan, P. A. Heiney, J. P. McCauley, Jr., and A. B. Smith, III, Phys. Rev. B 46, 2787 (1992).

${ }^{16}$ J. Simmerer, B. Glusen, W. Paulus, A. Kettner, P. Schuhmacher, D. Adam, K.-H. Etzbach, and K. Siemensmeyer, Adv. Math. 8, 815 (1996).

${ }^{17}$ S. Tasaka, T. Toyama, and N. Inagaki, Jpn. J. Appl. Phys., Part 1 33, 5838 (1994).

${ }^{18}$ J. N. Israelachvili, Intermolecular and Surface Forces, 2nd ed. (Academic, London, 1991).

${ }^{19}$ M. Ozaki, T. Sakurai, K. Sakamoto, M. Homma, and K. Yoshino, Jpn. J. Appl. Phys., Part 1 23, L175 (1984).

${ }^{20}$ N. W. Ashcroft and N. D. Mermin, Solid State Physics (Saunders College, Philadelphia, 1976).

${ }^{21}$ V. M. Fridkin, A. A. Grekov, P. V. Ionov, E. A. Savchenko, A. J. Rodin, and K. A. Verkjpvskaya, Ferroelectrics 8, 433 (1974).

${ }^{22}$ H. Sasabe, T. Nakayama, K. Kumazawa, S. Miyata, and E. Fukada, Polym. J. (Tokyo, Jpn.) 13, 967 (1981). 